American Journal of Infectious Diseases 1 (4): 156-161, 2005

ISSN 1553-6203

(C) 2005 Science Publications

\title{
Virus Dynamics in Vivo
}

\author{
Guang $\mathrm{Wu}$ and Shaomin Yan \\ Computational Mutation Project, DreamSciTech Consulting, 301, Building 12, Nanyou A-zone, Jiannan \\ Road, Shenzhen, Guangdong Province, CN-518054, China
}

\begin{abstract}
In this study, we model the interaction among antibody, virus, host cell, immune cell and normal cell as random collisions, because antibody and virus are unlikely to have efficient driving systems. Then we use the model II of waiting times to analyze the collision between antibody and virus with a certain number of normal cells, and use the Monte-Carlo simulation to analyze the random collision process with respect to different numbers of viruses, antibodies, normal, immune and host cells, and whether normal and host cells are fixed or free to move. Finally, we estimate the ratio of antibodies to viruses, which can prevent the host cells from virus infection at two endpoints, by means of the Monte Carlo simulation.
\end{abstract}

Key words: Antibody, dynamics, Monte Carlo simulation, Lanchester (Osipov) equation, random collision, Virus

\section{INTRODUCTION}

At current knowledge level, an antibody unlikely has any efficient driving system. This means that an antibody is more likely to encounter a virus by random collision. Actually even an antibody would have a very efficient driving system to overcome the blood circulation, but more importantly the immune detecting system should at first contact with the signal of a virus by chance, then the antibody can move with a definite direction.

Hence we need to consider the interaction between an antibody and a virus as a random collision from a probabilistic viewpoint. For the simplest example, there are three elements in our imaging system, a virus, a normal cell, and an antibody, so we have a 1 versus 2 system. The question is how many collisions are needed for the antibody to hit the virus. Our intuition with regard to this random collision process may suggest that the antibody would hit the virus at the second collision at the worst case, because the antibody spends the $1 / 2$ chance to randomly collide with the virus at the first collision. Our experience in daily life would tell us this intuition wrong because we could not expect to throw a ball randomly into the second hole if we have two holes and our first throw is in the first hole. Actually, the antibody always has the $1 / 2$ chance to randomly collide the virus at any collision, therefore the probability of random collision at the second collision is $0.5+1 / 2 \times$ the remained probability after the first collision, i.e. $1 / 2+1 / 2 \times(1-0.5)=0.75$, that says, the probability that the antibody hits the virus at the second collision is 0.75 . Thus, the antibody will hit the virus at the fifth collision if we set the 0.95 as the chance of random collision (Table 1). ${ }^{[1]}$ Or we can say that it needs five collisions for the antibody to hit the virus in this imaging system.

Table 1: Probability that an antibody hits the virus in 1 versus 2 system.

\begin{tabular}{llc}
\hline Collision & \multicolumn{1}{c}{ Calculation of collision probability } & Criterion \\
\hline $1^{\text {st }}$ & $0+1 / 2 \times(1-0)=0.5$ & $<0.95$ \\
$2^{\text {nd }}$ & $0.5+1 / 2 \times(1-0.5)=0.75$ & $<0.95$ \\
$3^{\text {rd }}$ & $0.75+1 / 2 \times(1-0.75)=0.875$ & $<0.95$ \\
$4^{\text {th }}$ & $0.875+1 / 2 \times(1-0.875)=0.9375$ & $<0.95$ \\
$5^{\text {th }}$ & $0.9375+1 / 2 \times(1-0.9375)=0.96875$ & $>0.95$ \\
$6^{\text {th }}$ & $0.96875+1 / 2 \times(1-0.96875)=0.984375$ & $>0.95$ \\
$\ldots$ & $\ldots$ & $\ldots$ \\
\hline
\end{tabular}

In the above consideration, the antibody hits, whereas the other two do not. This being the case, the virus and normal cell would be fixed. If both virus and normal cell are free to move, such as in blood, and each has the $1 / 2$ chance to randomly collide with the antibody, so how many collisions are needed for the antibody to hit the virus in this 1 versus 2 system? Our intuition may once again suggest that the antibody would hit the virus at the third collision because the virus can also hit the antibody with the same probability, so the number of collisions would be $5 / 2=2.5$. In fact, the situation is more complicated as the normal cell takes part in the collision process, and consumes the number of collisions. For example, if the

Corresponding Author: Guang Wu, MD, PhD, Computational Mutation Project, DreamSciTech Consulting, 301, Building 12, Nanyou A-zone, Jiannan Road, Shenzhen, Guangdong Province, CN-518054, China 
hitting proceeds in the following sequence, say, the antibody collides, the virus collides, the antibody collides, the normal cell collides, the normal cell collides, clearly the antibody will not hit the virus at the fifth collision in this particular sequence. We, however, can use the Monte Carlo simulation to minic this collision process by randomly choosing the collision sequence. Fig. 1 shows the distribution of 10,000 sets of simulations, and each set of simulations continues until the occurrence of collision between antibody and virus with $p>0.95$. Based on these 10,000 sets of simulations, we know that it needs $7.53 \pm 2.05$ collisions (mean $\pm \mathrm{SD}, \mathrm{n}=10,000, \min =5, \max =28$ ) for the antibody and virus to collide each other in this imagining system. Naturally, the more are the collisions needed for the antibody to hit the virus, the larger is the chance that the virus escapes from immune surveillance.

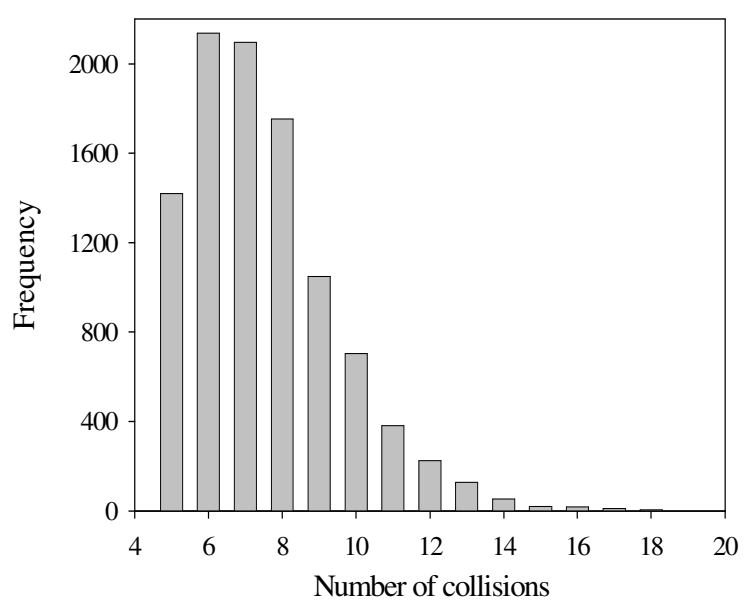

Fig. 1: Random collisions in 1 versus 2 system.

To have more practical feeling on the random collisions and its order of magnitude, we consider the chance of a leukocyte to hit an abnormal cell or a microbe in blood. In one-milliliter blood there are 4,300-10,800 leukocytes and 4,200,000-6,900,000 erythrocytes. On average one leukocyte has to monitor cells numbered between 639 cells $(6,900,000$ erythrocytes $) /(10,800$ leukocytes $)$ and 977 cells (4,200,000 erythrocytes)/(4,300 leukocytes). If there is an abnormal cell in the targeting range of a leukocyte, then we face the situation between 1 versus 640 system and 1 versus 978 system. Table 2 shows that the leukocyte will hit the abnormal cell between 1,916 and 2,929 collisions at the extremely best situation. This, though, is an unlikely case as only the leukocyte moves and others are fixed, it gives us a numerical concept. If all the cells are free to move, our Monte Carlo simulation indicates that it needs $612,367 \pm 1,916$ (mean $\pm \mathrm{SD}, \mathrm{n}=250, \min =607,948, \max =617,687)$ to $1,435,562 \pm 17,845 \quad($ mean $\pm \mathrm{SD}, \quad \mathrm{n}=250, \quad \min =$ $1,407,887, \quad \max =1,469,040)$ collisions for the leukocyte to hit its target.

The life span of most leukocytes is ranged from hours to years (neutrophils account for $54-75 \%$ of leukocytes with life span of 6 hours to a few days, eosinophils account $1-4 \%$ of leukocytes with life span of 8-12 days, basophils account for $0-1 \%$ of leukocytes with life span of a few hours to a few days, monocytes account for 2-8\% of leukocytes with life span of months, lymphocytes account for $25-40 \%$ of leukocytes with life span of days to years). A conceptual calculation of ratio of life span may help us estimate our random collision. If the life span is ranged to 100 days from 6 hours, the ratio is $(60 \times 60 \times 24 \times 100$ $\mathrm{sec}) /(60 \times 60 \times 6 \mathrm{sec})=(8,640,000 \mathrm{sec}) /(21,600 \mathrm{sec})=$ 400. The ratios in our Monte Carlo simulation are $(612,367$ collisions $) /(1,916$ collisions $)=320$ and $(1,435,562 \quad$ collisions $) /(2,929 \quad$ collisions $)=490$, therefore the ratio of leukocyte life span is in the same order of magnitude of ratio of random collisions.

Equally, the virus should hit its host cell by random collision because there is no driving system in virus too. Hence the interaction among antibody, virus, host cell, immune cell and normal cell is random collisions. In this study, we analyze the virus dynamics in vivo along this line of thought.

\section{METHODS}

Collision probability. The probabilistic model we used is the model II of waiting times, ${ }^{[2,3]}$ that is, the model continues randomly placing balls as long as a predefined hole remains empty. In our application, the probability that an antibody hits the virus is equal to accumulated probability + collision probability $\times(1-$ accumulated probability after last collision). We define $p>0.95$ as the probability of efficient collision between any two of antibody, virus, host cell, immune cell and normal cell, which means that the probability of missing collision is $<0.05$ as this probability is generally used in medical statistical practice. ${ }^{[1]}$

Monte Carlo simulation. The Monte Carlo simulation is used to analyze the random collision process with respect to different numbers of viruses, antibodies, normal, immune and host cells, and whether normal and host cells are fixed or free to move. A set of simulations continues until the occurrence of collision between our predefined elements such as antibody and virus with $p>0.95$. A hundred sets of simulations are conducted for each scenario. ${ }^{[8-10]}$ 
Table 2: Probability that a leukocyte hits an abnormal cell in 1 versus 640 system and 1 versus 978 system with $p>0.95$ as the criterion.

\begin{tabular}{lll}
\hline Collision & \multicolumn{1}{c}{ Calculation of probability of collision } \\
\hline & 1 versus 640 system & 1 versus 978 system \\
$1^{\text {st }}$ & $0+1 / 640 \times(1-0)=0.001563$ & $0+1 / 978 \times(1-0)=0.001022$ \\
$2^{\text {nd }}$ & $0.001563+1 / 640 \times(1-0.001563)=0.003123$ & $0.001022+1 / 978 \times(1-0.001022)=0.002044$ \\
$3^{\text {rd }}$ & $0.003123+1 / 640 \times(1-0.003123)=0.004680$ & $0.002044+1 / 978 \times(1-0.002044)=0.003064$ \\
$\ldots$ & $\ldots$ & $\ldots$ \\
$1916^{\text {th }}$ & $0.949940+1 / 640 \times(1-0.949940)=0.950018$ & $\ldots$ \\
$\ldots$ & $\ldots$ & $\ldots$ \\
$2929^{\text {th }}$ & $\ldots$ & $0.949983+1 / 978 \times(1-0.949983)=0.950034$ \\
\hline
\end{tabular}

\section{RESULTS AND DISCUSSION}

Collision between antibody and virus with a certain number of normal cells. The model we used in Introduction section is related to the collisions between an antibody and a virus with a certain number of normal cells. With $p>0.95$ as criterion, the minimal number of collisions can be regressed as follows: collisions $=3 \times$ (numbers of normal cells $)-3$. This means that the antibody needs additional 3 collisions to hit the virus when a normal cell is added into the collision system. Fig. 2 displays the pattern of this type of collision, and the intersection line between shadowed and unshadowed parts is the minimal number of collisions required for an antibody to hit the virus. The collision number should be located in the shadowed part if the virus and normal cells are free to move. The same reasoning is applied to the collision between virus and host cell with a certain number of normal cells, and the collision between virus and immune cell with a certain number of normal cells.

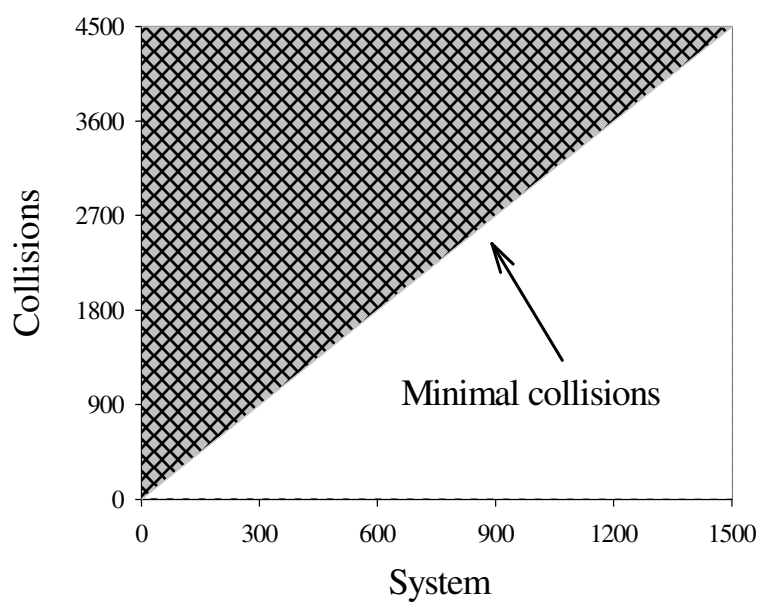

Fig. 2: Random collision between antibody and virus with a certain number of normal cells.

Collision in five-element system. As random collisions occur among antibody, host cell, immune cell, normal cell and virus, we can image a five-element system, which serves as the simplest dynamic system containing all the necessary elements for consideration just as the earth is considered as a point in the classical physics leading to the huge success in scientific history and changing our daily life. This five-element system contains an antibody, a host cell, an immune cell, a normal cell and a virus. Any real-life situation is a version from this imaging five-element system, and a deep analysis of collisions in this abstract five-element system can help us understand what happens in the real system.

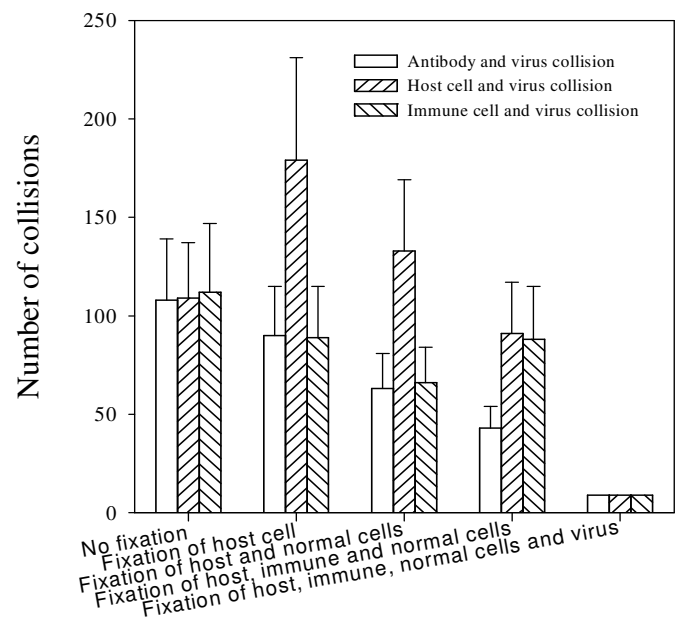

Fig. 3: Random collisions in five-element system with respect to whether or not an element is fixed.

In this five-element system, what we are actually interested in is the collisions of virus with antibody, host cell, and immune cell rather than with normal cell as well as the collisions among others. The only factor affecting the collision is whether or not an element is fixed (in tissue). Fig. 3 reveals several aspects with respect to whether or not an element is fixed using the Monte Carlo simulations: (i) the collisions between 
antibody and virus, between host cell and virus, and between immune cell and virus can equally occur if all five elements are free to hit others, (ii) the fixed element requires more collisions if others are free to hit, and (iii) the collision number is minimal if host cell, immune cell, normal cell and virus are fixed and only antibody is free to hit, which can be calculated using the regression equation.

It is somewhat unexpected that the collision number increases if an element is fixed, however this is reasonable when the collision is random and unaimed, because the fixed element loses the chance to actively hit others and can only be passively hit by others. The implication is that the host cell is more likely to be hit by a virus if the host cell is circulating in blood for example.

The unaimed fighting in mathematical language is the linear Lanchester (Osipov) equation for a single battle, ${ }^{[4,5]}$ which we used in medical settings in the past: $\left\{\begin{array}{l}\frac{d x}{d t}=-D x y \\ \frac{d y}{d t}=-A x y\end{array}\right\},{ }^{[6,7]}$ in our case $x$ is the number of attackers (viruses), $y$ is the number of defenders

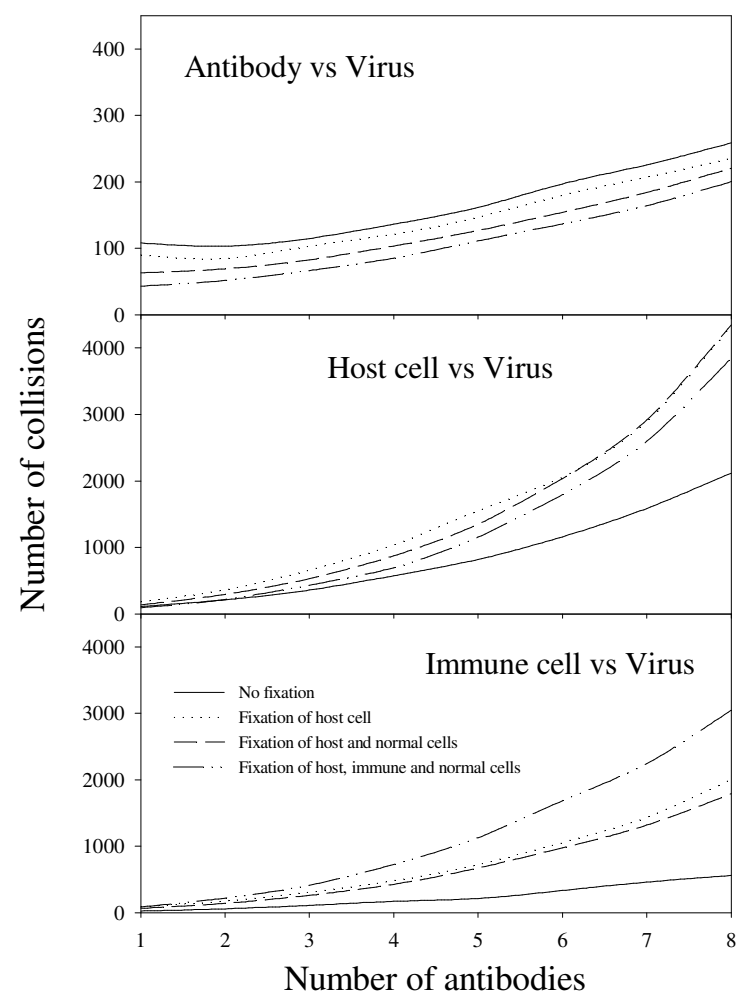

Fig. 4: Random collision with different numbers of antibodies with respect to whether or not an element is fixed. (antibodies), $A$ is the attacker's killing rate, $D$ is the defender's killing rate, $t$ is the time, and both attacker and defender are unaimed. However, the difficulty in the use of the Lanchester (Osipov) equation is that there are civilians (for example, normal cells) involved in the fighting, who play the role of human shield.

Reinforcement in antibody and virus. No matter what happens in collision, we will eventually face four scenarios, (i) the number of antibodies increases, in terms of the Lanchester (Osipov) equation, this is the battle with reinforcement on one side, $\left\{\begin{array}{l}\frac{d x}{d t}=-D x y \\ \frac{d y}{d t}=-A x y+\text { reinf orcement }\end{array}\right\} ;$ (ii) the number of viruses increases, this is $\left\{\begin{array}{l}\frac{d x}{d t}=-D x y+\text { reinf orcement } \\ \frac{d y}{d t}=-A x y\end{array}\right\} ;$ (iii) the numbers of both antibodies and viruses increase, this is

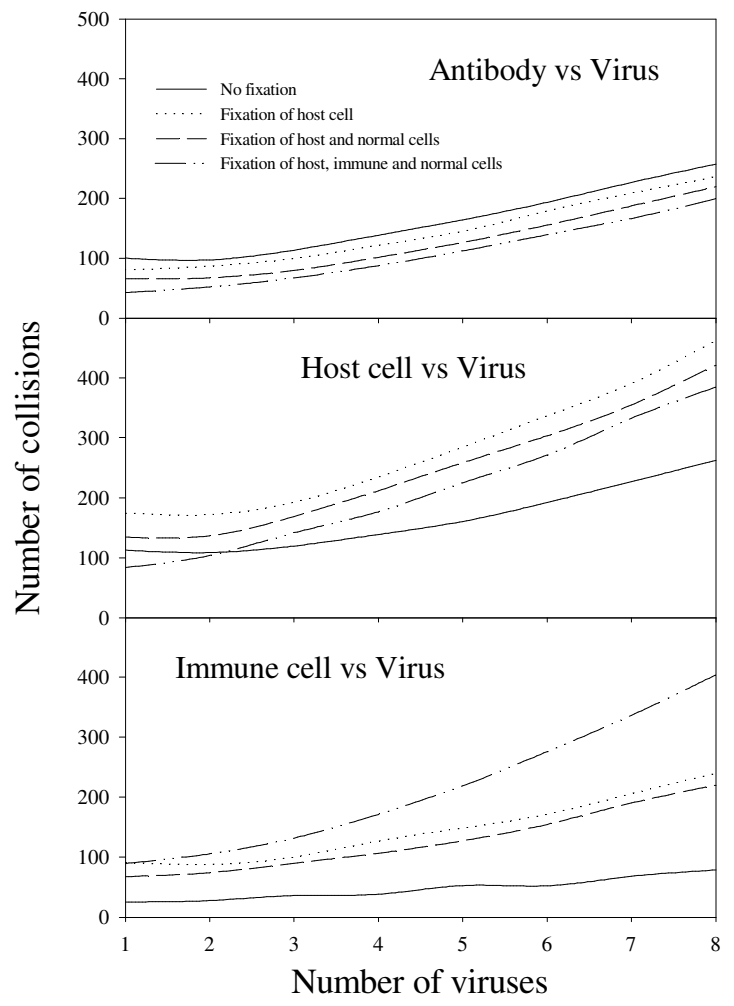

Fig. 5: Random collision with different numbers of viruses with respect to whether or not an element is fixed. 
the battle with reinforcement on both sides $\left\{\begin{array}{l}\frac{d x}{d t}=-D x y+\text { reinf orcement } \\ \frac{d y}{d t}=- \text { Axy + reinf orcement }\end{array}\right\} ;$ and (iv) the

battle is finished. Naturally the mathematical forms of reinforcements depend on the replication mechanism of virus and the production rate of antibody.

Fig. 4 enlightens the random collisions with different numbers of antibodies, which is the version of five-element system, that is, we keep the numbers of host cell, normal cell, immune cell and virus constant, and change the number of antibodies with respect to whether or not an element is fixed. Once again the randomness reveals something unexpected, that is, the number required for collision between antibody and virus increases with the increment of antibodies when the ratio of antibodies to virus is larger than 3 . This phenomenon is understandable, because the increase in antibodies will increase the collisions among themselves and decrease the collision probability. Although there is the possibility of occurring of simultaneous collisions, there should be a small interval between two collisions at extremely small time scale. Regarding whether or not an element is fixed in collision, the fixed element requires more collisions if others are free, which is similar to Fig. 3.

Fig. 5 illustrates the collisions with different numbers of viruses with respect to whether or not an element is fixed. In general, Fig. 5 is similar to Fig. 4, but the number of collisions is significantly lower in Fig. 5 than in Fig. 4. This is so because not only the collision between antibody and virus is meaningful, but also the collisions of virus with antibody, host cell and immune cell are all meaningful, thus the antibody has only $1 / 4$ chance to hit the virus in five-element system whereas the virus has $1 / 4$ chance to hit antibody, host cell and immune cell, respectively, in whole the virus has $3 / 4$ chance to hit them.

Hitting of $5 \%$ or $20 \%$ host cells by virus. We assume two endpoints in our Monte Carlo simulation: (i) 5\% host cells are hit by viruses, which should lead to statistically significant difference in comparison of numbers of host cells before and after virus infection, and (ii) $20 \%$ host cells are hit by viruses, which would result in disfunction in human body although no human data are available, and we assume so because the military history shows that on average one side would lose the battle if it loses $20 \%$ troops in the battle. ${ }^{[5]}$ In this context, we are interested in how many antibodies can prevent host cells from being hit by viruses with regard to 5\% and 20\% endpoints.

Generally, the more the viruses, the more the antibodies needed. However, we need to consider the relationship between host cells and antibodies. As usual, we can at first imagine that the hitting of viruses at host cell is similar to the placing of balls continues randomly into four types of holes, which represent antibody, immune, host and normal cells. A simple deduction would be that the more the antibodies, the smaller the probability that the virus randomly hits a host cell, that is, the larger the ratio of antibodies to host cells, the smaller the chance that the virus infects the host cell. Such a deduction is logical because a large number of troops should be allocated to defend a large city while a small number of troops should be deployed to defend a small village. Therefore the number of antibodies should increase with the increase of number of the host cells in order to defend them.
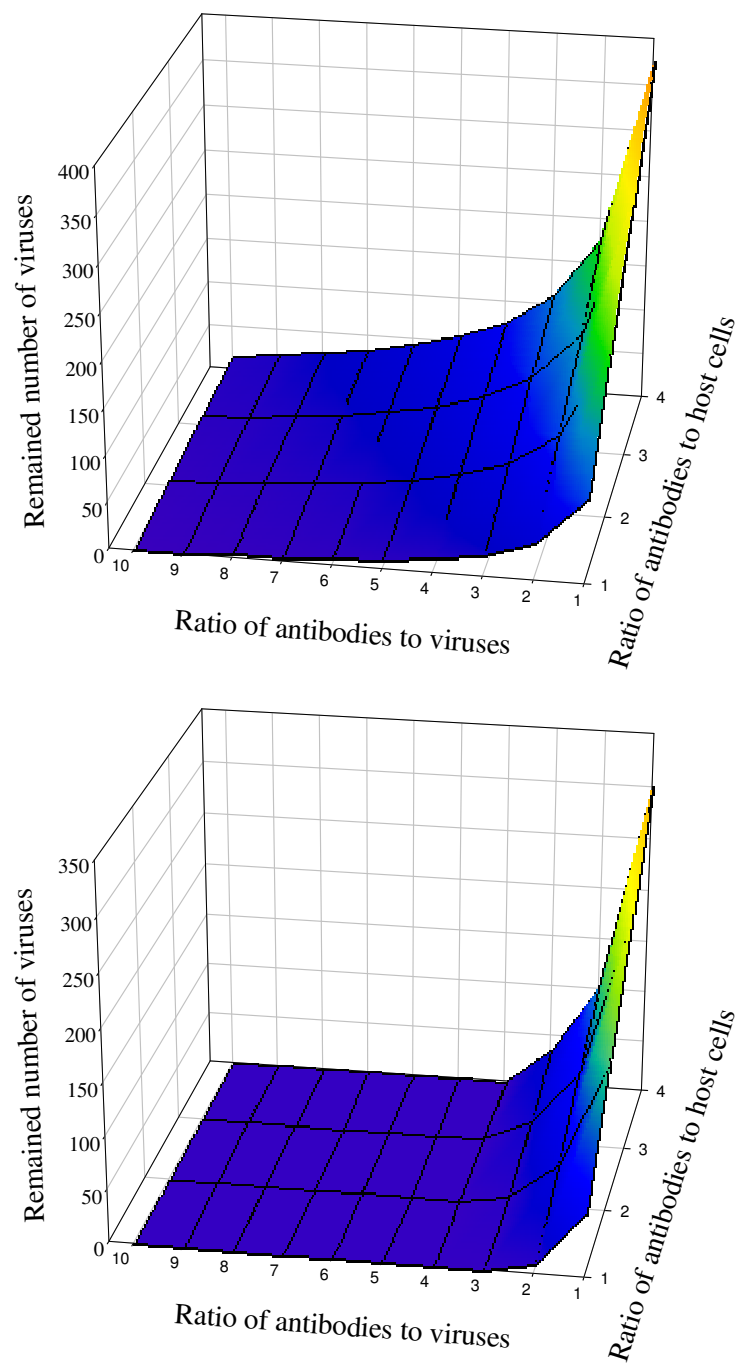

Fig. 6: Relationships among antibodies, host cells and viruses with regard to 5\% (the upper panel) and $20 \%$ (the lower panel) host cells hit by viruses.

Fig. 6 displays the relationships among antibodies, host cells and viruses found by the Monte Carlo simulations, where the $\mathrm{x}$ and $\mathrm{y}$ axis represent the ratios 
of antibodies to host cells and to viruses, respectively, while the $\mathrm{z}$ axis shows the remained number of viruses after hitting 5\% host cells (the upper panel) and $20 \%$ ones (the lower panel). Two general conclusions can be highlighted as follows: (i) virus infection (5\% host cells infected by viruses) can be avoid when the ratio of antibodies to viruses is larger than 10, and (ii) human body can be protected from disfunction (20\% host cells infected by viruses) when the antibodies overnumber viruses in 3 to 4 folds.

\section{ACKNOWLEDGEMENTS}

The authors wish to thank Dr RL Helmbold at US Army Concepts Analysis Agency, US Army's Center for Strategy and Force Evaluation, Bethesda, MD, USA for providing his reprints when the authors were at University of Udine, Udine, Italy, and Mr. Tianyu Wu at University of Udine for checking the references.

\section{REFERENCES}

1. Altman, D.G., S.M. Gore, M.J. Gardner, S.J. Pocock, 1983. Statistical guideline for contributors to medical journals. Br. Med. J., 286: 1489-93.

2. Feller, W., 1968. An Introduction to Probability Theory and its Applications, vol. I, third ed., New York: Wiley, pp. 47-50.

3. Wu, G. and S.M. Yan, 2002. Mathematical model of time needed for the immune system to detect and kill cancer cells in blood. Comp. Clin. Pathol. 11: 178-83.

4. Osipov, M., 1995. The influence of the numerical strength of engaged forces in their casualties. Translated by R.L. Helmbold, A.S. Rehm. Naval Res. Logistics 42: 435-90.

5. Helmbold, R.L. 1993. Osipov: The 'Russian Lanchester'. Eur. J. Oper. Res. 65: 278-88.

6. Wu, G., (22-Aug-2001) Modelling and prediction of the toxic effect, diarrhea, of EPO906 (epothilone B) in patients undergoing the clinical trials. Novartis Pharma AG, Internal Report, DMPK(CH) N00-2072-02, , Basel, Switzerland.

7. Wu, G. and S.M. Yan, 2002. Theoretical analysis of drug treatment in haematological disease using Lanchester (Osipov) linear law. Comp. Clin. Pathol. 11: 113-8.

8. Wu, G. 1998. Application of the queueing theory with Monte Carlo simulation to inhalation toxicology. Arch. Toxicol. 72: 330-5.

9. Wu, G. 1998. Application of queueing theory with Monte Carlo simulation to the study of the intake and adverse effects of ethanol. Alcohol Alcohol. 33: 519-27.

10. Wu, G. and S.M. Yan, 2001. Estimation of the rate of arrivals of ions at a single-channel. J. Biochem. Mol. Biol. Biophy. 5: 505-11. 